\title{
Makarov's principle for the Bloch unit ball
}

\author{
Oleg Ivrii and Ilgiz Kayumov \\ February 18, 2017
}

\begin{abstract}
Makarov's principle relates three characteristics of Bloch functions that resemble the variance of a Gaussian: asymptotic variance, the constant in Makarov's law of iterated logarithm and the second derivative of the integral means spectrum at the origin. While these quantities need not be equal in general, we show that the universal bounds agree if we take the supremum over the Bloch unit ball. For the supremum (of either of these quantities), we give the estimate $\Sigma_{\mathcal{B}}^{2}<\min \left(0.9, \Sigma^{2}\right)$, where $\Sigma^{2}$ is the analogous quantity associated to the unit ball in the $L^{\infty}$ norm on the Bloch space. This improves on the upper bound in Pommerenke's estimate $0.685^{2}<\Sigma_{\mathcal{B}}^{2} \leq 1$.
\end{abstract}

\section{Introduction}

The Bloch space consists of analytic functions in the unit disk for which

$$
\|b\|_{\mathcal{B}}:=\sup _{z \in \mathbb{D}}\left(1-|z|^{2}\right)\left|b^{\prime}(z)\right|<\infty
$$

while a function $b_{0}$ belongs to the little Bloch space $\mathcal{B}_{0}$ if

$$
\lim _{|z| \rightarrow 1^{-}}\left(1-|z|^{2}\right)\left|b_{0}^{\prime}(z)\right|=0
$$

Makarov's principle [11] is concerned with three characteristics of functions $b \in \mathcal{B} / \mathcal{B}_{0}$ that measure the growth of $b$ near the unit circle: 
- The asymptotic variance

$$
\sigma^{2}(b)=\limsup _{r \rightarrow 1} \frac{1}{2 \pi|\log (1-r)|} \int_{|z|=r}|b(z)|^{2}|d z| .
$$

- The LIL constant

$$
C_{\mathrm{LIL}}(b)=\underset{\theta \in[0,2 \pi)}{\operatorname{ess} \sup }\left\{\limsup _{r \rightarrow 1} \frac{\left|b\left(r e^{i \theta}\right)\right|}{\sqrt{\log \frac{1}{1-r} \log \log \log \frac{1}{1-r}}}\right\} .
$$

- The integral means spectrum

$$
\beta_{b}(\tau)=\limsup _{r \rightarrow 1} \frac{1}{|\log (1-r)|} \cdot \log \int_{|z|=r}\left|e^{\tau b(z)}\right||d z|, \quad \tau \in \mathbb{C} .
$$

The above quantities are unrelated for general Bloch functions, see [4, 13] for interesting examples. Nevertheless, when one takes the supremum over natural classes of Bloch functions, the universal bounds coincide. In this paper, we prove Makarov's principle for the Bloch unit ball:

\section{Theorem 1.1.}

$$
\Sigma_{\mathcal{B}}^{2}:=\sup _{\|b\|_{\mathcal{B}} \leq 1} \sigma^{2}(b)=\sup _{\|b\|_{\mathcal{B}} \leq 1} C_{\mathrm{LIL}}^{2}(b)=\lim _{\tau \rightarrow 0} \frac{4}{|\tau|^{2}} \cdot \sup _{\|b\|_{\mathcal{B}} \leq 1} \beta_{b}(\tau) .
$$

We also give two different upper bounds for $\Sigma_{\mathcal{B}}^{2}$. The first upper bound is an explicit estimate, while the second upper bound is in terms of an analogous quantity associated to the unit ball of $\mathcal{B} / \mathcal{B}_{0}$ equipped with the " $L^{\infty}$ norm."

\section{Theorem 1.2.}

$$
\Sigma_{\mathcal{B}}^{2}<\min \left(0.9, \Sigma^{2}\right) .
$$

Previously, it was known that $C_{\mathrm{LIL}}(b) \leq\|b\|_{\mathcal{B}}$ which was first established by Pommerenke [20] in 1985 who used an iterative scheme involving Hardy's identity. Two other proofs of this fact appeared since then: Bañuelos [3] came up with a clever argument based on an $L^{\infty}$ estimate for the Littlewood-Paley $g_{*}$ function while Lyons [14] used hyperbolic Brownian motion. We can also mention the efforts of 
Przytycki [22] who proved the weaker statement $C_{\mathrm{LIL}}(b) \leq \frac{16}{\log 2}\|b\|_{\mathcal{B}}$ by emulating a method of Philipp and Stout for lacunary trigonometric series, as well as Makarov's original result [15] which says that $C_{\mathrm{LIL}}(b) \leq C\|b\|_{\mathcal{B}}$ holds with some constant $C>0$. Nevertheless, the question whether " $C_{\mathrm{LIL}}(b) \leq\|b\|_{\mathcal{B}}$ " was sharp remained open. The above theorem answers this question in the negative.

For the lower bound, Pommerenke gave an example of a Bloch function with $C_{\text {LIL }}(b) \leq 0.685\|b\|_{\mathcal{B}}$, see [21, Theorem 8.10].

\subsection{The $L^{\infty}$ norm on the Bloch space}

The original statement of Makarov's principle from [11] deals with another unit ball which is more natural from the point of view of quasiconformal geometry. There, $\mathcal{B} / \mathcal{B}_{0}$ is equipped with the " $L^{\infty}$ norm" coming from the representation $B=P\left(L^{\infty}\right)$ via the Bergman projection

$$
P \mu(z)=\frac{1}{\pi} \int_{\mathbb{D}} \frac{\mu(w)}{(1-z \bar{w})^{2}}|d w|^{2} .
$$

That is,

$$
\|b\|_{\mathcal{B} / \mathcal{B}_{0}, \infty}:=\inf _{P \mu \sim b}\|\mu\|_{\infty}
$$

where the infimum is taken over all $\mu \in L^{\infty}(\mathbb{D})$ such that $P \mu=b+b_{0}$ with $b_{0} \in \mathcal{B}_{0}$. When one takes the supremum over the $\left(\mathcal{B} / \mathcal{B}_{0}, \infty\right)$ unit ball, one obtains a different constant:

$$
\Sigma^{2}:=\sup _{\|\mu\|_{\infty} \leq 1} \sigma^{2}(P \mu)=\sup _{\|\mu\|_{\infty} \leq 1} C_{\mathrm{LIL}}^{2}(P \mu)=\lim _{\tau \rightarrow 0} \frac{4}{|\tau|^{2}} \cdot \sup _{\|\mu\|_{\infty} \leq 1} \beta_{P \mu}(\tau) .
$$

The quantitity $\Sigma^{2}$ is naturally related to the problem of dimensions of quasicircles. If $D(k)$ is the maximal dimension of a $k$-quasicircle, then according to [1, 8, 10],

$$
\begin{gathered}
D(k)=1+\Sigma^{2} k^{2}+o\left(k^{2}\right), \quad 0<k<1, \\
0.879<\Sigma^{2}<1 .
\end{gathered}
$$

The proof of Theorem 1.1 uses fractal approximation techniques and is quite similar to that of (1.5), but requires some modifications which we describe in this paper. In 
fact, the argument is applicable with any reasonable norm on $\mathcal{B} / \mathcal{B}_{0}$, for instance,

$$
\|b\|_{\mathcal{B} / \mathcal{B}_{0}, m}=\limsup _{|z| \rightarrow 1}\left(1-|z|^{2}\right)^{m}\left|b^{(m)}(z)\right|, \quad m \geq 2
$$

lead to the constants $\Sigma_{\mathcal{B}, m}^{2}$. The details will be given in Section 3 ,

\subsection{Why $\Sigma_{\mathcal{B}}^{2}<1$ ?}

We now describe the idea behind the bound " $\Sigma_{\mathcal{B}}^{2} \leq 0.9$." Suppose $b$ is a Bloch function of norm 1. Since $b$ is a holomorphic function, it is reasonable to expect that the Bloch quotient $\left|2 b^{\prime} / \rho\right|=\left|b^{\prime}(z)\right|\left(1-|z|^{2}\right)$ is strictly less than 1 on average, where $\rho(z)=\frac{2}{1-|z|^{2}}$ is the density of the hyperbolic metric on the unit disk. One way to make this precise is to say that

$$
\alpha(R):=\sup _{\|b\|_{\mathcal{B}} \leq 1}\left[\sup _{B} f_{B}\left|\frac{2 b^{\prime}}{\rho}(z)\right|^{2} \rho^{2}|d z|^{2}\right]<1,
$$

where the inner supremum is taken over all balls $B \subset \mathbb{D}$ of hyperbolic radius $R$. Here, the notation $f \ldots \rho^{2}|d z|^{2}$ suggests that we consider the average in the hyperbolic metric. We will later give a quantitative estimate for $\alpha(R)$, but in order to prove (1.7), the following observation is sufficient:

Lemma 1.1. Suppose $b \in \mathcal{B}$ is a Bloch function with $\|b\|_{\mathcal{B}}=1$. There exists $R, S>$ 0 such that any ball $B=B_{\text {hyp }}(z, R) \subset \mathbb{D}$ of hyperbolic radius $R$ contains a ball $B_{\mathrm{hyp}}(\zeta, S)$ on which the Bloch quotient is less than $1 / 2$.

Proof. Since the Bloch quotient is invariant under automorphisms of the disk, it is enough to consider the case when $B$ is centered at the origin. If the lemma were false, then by the Lipschitz property of Bloch functions, there would exist a sequence of functions $b_{n}$ in the Bloch unit ball with $\left|2 b_{n}^{\prime} / \rho\right|>1 / 10$ on $B_{\text {hyp }}(0, n)$. A normal families argument would produce a Bloch function $b$ for which the Bloch quotient was strictly bounded away from 0 on the entire disk. This would contradict the maximum modulus principle applied to $1 / b^{\prime}$.

In Section 4, we will show that $\alpha(R)$ dominates the asymptotic variance and find a value of $R$ for which $\alpha(R) \leq 0.9$. 


\section{Lemma 1.2.}

$$
\sup _{\|b\|_{\mathcal{B}}=1} \sigma^{2}(b) \leq \inf _{R>0} \alpha(R)
$$

Theorem 1.1 implies that the right hand side of (1.8) also bounds the LIL constant and the quadratic behaviour of the integral means spectrum at the origin.

\subsection{An application to harmonic measure}

To conclude the introduction, we apply Makarov's principle for the Bloch unit ball to study metric properties of harmonic measure of simply-connected domains. Let $\mathbf{S}$ denote the collection of conformal maps $f: \mathbb{D} \rightarrow \mathbb{C}$ satisfying $f(0)=0$ and $f^{\prime}(0)=1$. The Becker class $\mathbf{S}_{\mathrm{B}} \subset \mathbf{S}$ consists of conformal maps for which $\left\|\log f^{\prime}\right\|_{\mathcal{B}} \leq 1$. According to Becker's univalence criterion, it is in bijection with functions in the Bloch unit ball with $b(0)=0$. A theorem of Makarov [16], [7, Theorem VIII.2.1] shows:

Corollary 1.1. (i) Let $f \in \mathbf{S}_{\mathrm{B}}$ be a function in the Becker class, $\Omega=f(\mathbb{D})$ be the image of the unit disk and $z_{0}$ be a point in $\Omega$. The harmonic measure $\omega_{z_{0}}$ on $\partial \Omega$ as viewed from $z_{0}$ is absolutely continuous with respect to the Hausdorff measure $\Lambda_{h(t)}$,

$$
h(t)=t \exp \left\{C \sqrt{\log \frac{1}{t} \log \log \log \frac{1}{t}}\right\}, \quad 0<t<10^{-7}
$$

for any $C \geq C_{\mathrm{LIL}}\left(\log f^{\prime}\right)$. In particular, $C=\sqrt{\Sigma_{\mathcal{B}}^{2}}$ works.

(ii) Conversely, if $C<\sqrt{\Sigma_{\mathcal{B}}^{2}}$, there exists a conformal map in $\mathbf{S}_{\mathrm{B}}$ for which $\omega_{z_{0}} \perp \Lambda_{h(t)}$.

The above corollary has a surprising consequence. As discussed in [10],

$$
\sup _{f \in \mathbf{S}} \sigma^{2}\left(\log f^{\prime}\right) \geq \Sigma^{2}>\Sigma_{\mathcal{B}}^{2}=\sup _{f \in \mathbf{S}_{\mathrm{B}}} \sigma^{2}\left(\log f^{\prime}\right)
$$

which shows that functions in the Becker class are rather tame. For more bounds on $\sup _{f \in \mathbf{S}} \sigma^{2}\left(\log f^{\prime}\right)$, we refer the reader to the works [12, 9, 1 . 


\section{Acknowledgements}

The first author was supported by the Academy of Finland, project nos. 271983 and 273458. The second author was supported by the RFBR and the government of the Republic of Tatarstan, project nos. 14-01-00351 and 15-41-02433.

\section{The Bergman projection}

As mentioned in the introduction, the Bergman projection takes $L^{\infty}(\mathbb{D})$ to the Bloch space. The estimate $\Sigma_{\mathcal{B}}^{2}<\Sigma^{2}$ is immediate from the following representation which is interesting in its own right:

Lemma 2.1. The Bergman projection $P: L^{\infty}(\mathbb{D}) \rightarrow \mathcal{B} / \mathcal{B}_{0}$ is surjective. Any $b \in \mathcal{B}$ may be represented as

$$
b=P \mu+b_{0}, \quad \text { with } \quad\|\mu\|_{\infty} \leq C\|b\|_{\mathcal{B}}, \quad b_{0} \in \mathcal{B}_{0}
$$

Furthermore, the optimal constant $C$ in 2.1) is strictly less than 1.

Proof. For a Bloch function $b \in \mathcal{B}$, set

$$
\mu_{b}(z):=(1 / \bar{z}) \cdot 2 b^{\prime} / \rho
$$

The reader may notice that $\mu_{b}$ is not bounded near the origin, however, only the asymptotic bound $\lim \sup _{|z| \rightarrow 1}\left|\mu_{b}(z)\right| \leq\|b\|_{\mathcal{B}}$ is essential here. We claim that $P \mu_{b}(z)=$ $b(z)-b(0)$. For this purpose, we consider the reproducing formula

$$
f(z)=\frac{2}{\pi} \int_{\mathbb{D}} \frac{f(w)\left(1-|w|^{2}\right)}{(1-z \bar{w})^{3}}|d w|^{2}
$$

of the weighted Bergman space $A_{1}^{2}$ with norm

$$
\|f(z)\|_{A_{1}^{2}}^{2}=\frac{2}{\pi} \int_{\mathbb{D}}|f(z)|^{2}\left(1-|z|^{2}\right)|d z|^{2},
$$

for instance see [23, Theorem 2.7]. By considering dilates $f(r z)$ and taking $r \rightarrow 1^{-}$, it follows that 2.3 holds for all holomorphic functions $f$ for which

$$
\|f\|_{A_{1}^{\infty}}:=\sup _{z \in \mathbb{D}}|f(z)|\left(1-|z|^{2}\right)<\infty .
$$


This allows us to take $f=b^{\prime}$ which gives $\left(P \mu_{b}\right)^{\prime}=b^{\prime}$, thus $P \mu_{b}$ and $b$ agree up to an additive constant. To evaluate this constant, note that $P \mu_{b}(0)=0$ by the definition of the Bergman projection (1.4). This proves the claim.

To proceed further, we use duality considerations. Without loss of generality, we can assume that $\|b\|_{\mathcal{B}}=1$ and $b(0)=0$. As is well known, e.g. see [23, Theorem 3.17] or [8], the Bloch space equipped with the $L^{\infty}$ norm $\|b\|_{\mathcal{B}, \infty}:=\inf _{P \nu=b}\|\nu\|_{\infty}$ is the isometric dual of the Bergman space $A^{1}$ with respect to the pairing

$$
\langle b, g\rangle=\lim _{r \rightarrow 1} \frac{1}{\pi} \int_{\mathbb{D}} b(z) \overline{g(r z)}|d z|^{2}, \quad b \in \mathcal{B}, g \in A^{1} .
$$

Since the Bergman projection is self-adjoint, the above duality implies

$$
\inf _{P \nu=b}\|\nu\|_{\infty}=\left.\sup _{g}\left|\int_{\mathbb{D}} \mu_{b}(z) \overline{g(z)}\right| d z\right|^{2}\left|, \quad \int_{\mathbb{D}}\right| g(z) \mid=1 .
$$

Readers familiar with asymptotic Teichmüller spaces (see [6, Chapter 14.10]) will recognize that the infimum of $\|\nu\|_{\infty}$ over $\nu \in L^{\infty}(\mathbb{D})$ satisfying $P \nu=b+b_{0}$ with $b_{0} \in \mathcal{B}_{0}$ and $b_{0}(0)=0$ is given by

$$
\inf _{P \nu=b+b_{0}}\|\nu\|_{\infty}=\left.\sup _{\left\{g_{n}\right\}} \limsup _{n \rightarrow \infty}\left|\int_{\mathbb{D}} \mu_{b}(z) \overline{g_{n}(z)}\right| d z\right|^{2} \mid,
$$

where the supremum is taken over all degenerating Hamilton sequences $\left\{g_{n}\right\} \subset A^{1}$, i.e. sequences with $\int_{\mathbb{D}}\left|g_{n}\right|=1$ for which $g_{n} \rightarrow 0$ uniformly on compact subsets of the disk. A normal families argument and Lemma 1.1 show that a definite proportion of the mass of $\left|g_{n}\right|$ is "wasted" on the set where $\left|2 b^{\prime} / \rho\right|<1 / 2$. Therefore, the right hand side of $(2.5)$ is bounded by a constant strictly less than 1 .

Remark. One can compare Lemma 2.1 to a result of Perälä [19] which says that the operator seminorm $\|P\|_{L^{\infty} \rightarrow \mathcal{B}}=8 / \pi$. By contrast, the optimal constant $C$ in (2.1) is not known. 


\subsection{Working in the upper half-plane}

While it is simpler to visualize the duality arguments in the unit disk, the upper half-plane is a more natural setting for the fractal approximation techniques. We are therefore led to consider the Bloch space $\mathcal{B}(\mathbb{H})$ which consists of holomorphic functions on $\mathbb{H}$ with

$$
\|b\|_{\mathcal{B}(\mathbb{H})}=\sup _{z \in \mathbb{H}} 2 y \cdot\left|b^{\prime}(z)\right|<\infty .
$$

Instead of using the Bergman projection, we prefer to represent Bloch functions in $\mathbb{H}$ via the modified Beurling transform $\mathcal{S}^{\#}: L^{\infty}(\overline{\mathbb{H}}) \rightarrow \mathcal{B}(\mathbb{H})$,

$$
\mathcal{S}^{\#} \mu(z)=-\frac{1}{\pi} \int_{\overline{\mathbb{H}}} \mu(w)\left[\frac{1}{(w-z)^{2}}-\frac{1}{w(w-1)}\right]|d w|^{2},
$$

which includes the term $-\frac{1}{w(w-1)}$ to guarantee convergence. We often abuse notation and write

$$
"(\mathcal{S} \mu)^{\prime}(z) ":=\left(\mathcal{S}^{\#} \mu\right)^{\prime}(z)=-\frac{2}{\pi} \int_{\mathbb{\mathbb { H }}} \frac{\mu(w)}{(w-z)^{3}}|d w|^{2} .
$$

In this setting, (2.2) becomes

$$
\mu_{b}=2 i \cdot \overline{b^{\prime}(\bar{z})} / \rho_{\overline{\mathbb{H}}}, \quad \mathcal{S}^{\#} \mu_{b}=b+C .
$$

\subsection{Locality of the Beurling transform}

For our purposes, the most important property of the Beurling transform is that it is local in nature [10, Section 4]:

Lemma 2.2. (i) Suppose $\mu$ is a Beltrami coefficient supported on the lower half-plane with $\|\mu\|_{\infty} \leq 1$. Then, $\left|\left(2(\mathcal{S} \mu)^{\prime} / \rho_{\mathbb{H}}\right)(z)\right| \leq 8 / \pi$.

(ii) For any $\varepsilon>0$, there exists $R>0$ sufficiently large so that if the hyperbolic distance $d_{\overline{\mathbb{H}}}(\bar{z}, \operatorname{supp} \mu) \geq R$ then $\left|\left(2(\mathcal{S} \mu)^{\prime} / \rho_{\mathbb{H}}\right)(z)\right| \leq \varepsilon$.

In particular, if $\mu_{1}$ and $\mu_{2}$ are two Beltrami coefficients on $\overline{\mathbb{H}}$ with $\left\|\mu_{i}\right\|_{\infty} \leq 1$, $i=1,2$ that agree on a ball $B_{\text {hyp }}(\bar{z}, R) \subset \overline{\mathbb{H}}$ with $R$ large, then

$$
\left|\frac{2\left(\mathcal{S} \mu_{1}\right)^{\prime}}{\rho_{\mathbb{H}}}(z)-\frac{2\left(\mathcal{S} \mu_{2}\right)^{\prime}}{\rho_{\mathbb{H}}}(z)\right| \leq 2 \varepsilon .
$$




\subsection{Boxes and grids}

By a box in the upper half-plane, we mean a rectangle whose sides are parallel to the coordinate axes, with the bottom side located above the real axis. Boxes naturally arise in grids. One natural collection of grids are the $n$-adic grids $\mathscr{G}_{n}$, defined for integer $n \geq 2$. An $n$-adic interval $I \subset \mathbb{R}$ is an interval of the form $I_{j, k}=\left[j \cdot n^{-k},(j+1) \cdot n^{-k}\right]$. To an $n$-adic interval $I$, we associate the $n$-adic box

$$
\square_{I}=\left\{w: \operatorname{Re} w \in I, \operatorname{Im} w \in\left[n^{-1}|I|,|I|\right]\right\} .
$$

It is easy to see that the boxes $\square_{I_{j, k}}$ with $j, k \in \mathbb{Z}$ have disjoint interiors and their union is $\mathbb{H}$.

If $\mu$ is a Beltrami coefficient supported on the lower half-plane, we say that $\mu$ is periodic with respect to a grid $\mathscr{G}$ (or rather with respect to $\overline{\mathscr{G}}$ ) if for any two boxes $\bar{B}_{1}, \bar{B}_{2} \in \overline{\mathscr{G}}$, we have $\left.\mu\right|_{\bar{B}_{1}}=L^{*}\left(\left.\mu\right|_{\bar{B}_{2}}\right)$, where $L(z)=a z+b, a>0, b \in \mathbb{R}$, is the affine map that takes $\bar{B}_{1}$ to $\bar{B}_{2}$. We remind the reader that when computing the pullback, Beltrami coefficients are viewed as $(-1,1)$-forms.

Given $\mu$ defined on a box $\bar{B}$, and a grid $\overline{\mathscr{G}}$ containing $\bar{B}$, there exists a unique periodic Beltrami coefficient $\mu_{\text {per }}$ which agrees with $\mu$ on $\bar{B}$. As discussed in [11, for Bloch functions $b=\mathcal{S}^{\#} \mu$ with periodic $\mu$, the three characteristics in Makarov's principle are equal.

\subsection{An isoperimetric property of the metric $|d z|^{2} / y$}

The metric $|d z|^{2} / y$ will play an important role in the work. The crucial feature that makes the periodization arguments work is the following isoperimetric property: if $S>0$ is held fixed, then

$$
\frac{\operatorname{Area}\left(\partial_{S} \square,|d z|^{2} / y\right)}{\operatorname{Area}\left(\square,|d z|^{2} / y\right)} \rightarrow 0, \quad \square \in \mathscr{G}_{n}, \quad n \rightarrow \infty,
$$

where $\partial_{S} \square:=\left\{z \in \square: d_{\mathbb{H}}(z, \partial \square)<S\right\}$.

Combining the isoperimetric property of $|d z|^{2} / y$ with 2.9$)$, we see that if $\mu_{1}=\mu_{2}$ agree on the reflection $\square$ of a box $\square \in \mathcal{G}_{n}$, then the difference

$$
\left.\left|f_{\square}\right| \frac{2\left(\mathcal{S} \mu_{1}\right)^{\prime}}{\rho_{\mathbb{H}}}(z)\right|^{2} \frac{|d z|^{2}}{y}-f_{\square}\left|\frac{2\left(\mathcal{S} \mu_{2}\right)^{\prime}}{\rho_{\mathbb{H}}}(z)\right|^{2} \frac{|d z|^{2}}{y} \mid=o(1), \quad \text { as } n \rightarrow \infty .
$$


Here, we have used the elementary identity $\left.|| a\right|^{2}-|b|^{2}|\leq| a-b|\cdot| a+b \mid$ and Lemma 2.2 (i).

\section{Box Lemma}

For a Bloch function $b \in \mathcal{B}(\mathbb{H})$, its asymptotic variance is given by

$$
\begin{aligned}
\sigma_{[0,1]}^{2}(b) & =\limsup _{y \rightarrow 0^{+}} \frac{1}{|\log y|} \int_{0}^{1}|b(x+i y)|^{2} d x, \\
& =\limsup _{h \rightarrow 0^{+}} \frac{1}{|\log h|} \int_{h}^{1} \int_{0}^{1}\left|\frac{2 b^{\prime}(x+i y)}{\rho_{\mathbb{H}}}\right|^{2} \frac{|d z|^{2}}{y} .
\end{aligned}
$$

The equivalence of the two definitions is due to McMullen [18, Section 6], for a purely upper half-plane proof, see [11]. It is not difficult to show that the maximum asymptotic variance over the unit ball in $\mathcal{B}(\mathbb{H})$ coincides with the unit disk version:

$$
\Sigma_{\mathcal{B}}^{2}=\sup _{\|b\|_{\mathcal{B}(\mathbb{H})} \leq 1} \sigma_{[0,1]}^{2}(b) .
$$

The same is true for the other characteristics of Bloch functions from the introduction. One can prove this by precomposing Bloch functions $b \in \mathcal{B}$ with the exponential $\xi(w)=e^{2 \pi i w}$ like in [10, Section 3]. We leave the details to the reader.

The proof of Makarov's principle for the Bloch unit ball rests on the following Box Lemma:

Lemma 3.1. (i) Given $\varepsilon>0$, if $n \geq n(\varepsilon)$ is sufficiently large, then for any Bloch function $b$ with $\|b\|_{\mathcal{B}(\mathbb{H})} \leq 1$ and $\square \in \mathscr{G}_{n}$,

$$
f_{\square}\left|\frac{2 b^{\prime}}{\rho_{\mathbb{H}}}(z)\right|^{2} \frac{|d z|^{2}}{y}<\Sigma_{\mathcal{B}}^{2}+\varepsilon .
$$

(ii) Conversely, for $n \geq n(\varepsilon)$ sufficiently large, there exists a Bloch function $b$ with $\|b\|_{\mathcal{B}(\mathbb{H})} \leq 1$, which satisfies

$$
f_{\square}\left|\frac{2 b^{\prime}}{\rho_{\mathbb{H}}}(z)\right|^{2} \frac{|d z|^{2}}{y}>\Sigma_{\mathcal{B}}^{2}-\varepsilon
$$

on every box $\square \in \mathscr{G}_{n}$. Furthermore, $b$ may be taken of the form $b=\mathcal{S}^{\#} \mu$ for some Beltrami coefficient $\mu$ with $|\mu| \leq \chi_{\overline{\mathbb{H}}}$ that is periodic with respect to the $n$-adic grid. 
With the help of Lemma 3.1, the proof of Theorem 1.1 can be completed using the scheme laid out in [11]:

1. Following Makarov [17], a Bloch function $b \in \mathcal{B}(\mathbb{H})$ defines an $n$-adic martingale on $[0,1]$. Namely, for an $n$-adic interval $I \subset[0,1]$, one can define

$$
B_{I}:=\lim _{h \rightarrow 0^{+}} \frac{1}{|I|} \int_{I+i h} b(x+i h) d x
$$

so that if $I_{1}, I_{2}, \ldots, I_{n}$ are the $n$-adic children of $I$, then $B_{I}=(1 / n) \sum_{j=1}^{n} B_{I_{j}}$.

2. Box averages (3.3) describe the local variation of this martingale. Set

$$
\operatorname{Var}_{I} B:=\frac{1}{n} \sum_{j=1}^{n}\left|B_{I_{j}}-B_{I}\right|^{2}
$$

An argument involving Green's formula on $\square_{I}$ shows that

$$
\frac{\operatorname{Var}_{I} B}{\log n}=f_{\square_{I}}\left|\frac{2 b^{\prime}}{\rho_{\mathbb{H}}}(z)\right|^{2} \frac{|d z|^{2}}{y}+\mathcal{O}\left(\|b\|_{\mathcal{B}(\mathbb{H})}^{2} / \sqrt{\log n}\right) .
$$

3. The local variance controls the three characteristics in Makarov's principle. Let $m=\inf _{I} \frac{\operatorname{Var}_{I} B}{\log n}$ and $M=\sup _{I} \frac{\operatorname{Var}_{I} B}{\log n}$. One can show that the three characteristics in Makarov's principle are pinched between $m$ and $M$. The key point is that the characteristics can be defined in terms of the martingale $B$, so the problem is purely combinatorial.

Proof of Lemma 3.1. (i) Assume for the sake of contradiction that there is a box $\square \subset \mathbb{H}$ and a function $b$ in the Bloch unit ball for which

$$
f_{\square}\left|\frac{2 b^{\prime}}{\rho_{\mathbb{H}}}(z)\right|^{2} \frac{|d z|^{2}}{y}>\Sigma_{\mathcal{B}}^{2}+\varepsilon .
$$

We can choose a Beltrami coefficient $\mu$ with $\mu \leq \chi_{\overline{\mathbb{H}}}$ and $\mathcal{S}^{\#} \mu=b+C$, for instance $\mu=2 i \cdot \overline{b^{\prime}(\bar{z})} / \rho_{\overline{\mathbb{H}}}$ will do, cf. 2.8. We then form the Beltrami coefficient $\mu_{\mathrm{per}} \leq \chi_{\overline{\mathbb{H}}}$ by restricting $\mu$ to $\bar{\square}$ and periodizing with respect to $\overline{\mathscr{G}_{n}}$, that is, on $\bar{\square}_{j} \in \overline{\mathscr{G}_{n}}$, we 
define $\mu_{\text {per }}=L_{j}^{*} \mu$, where $L_{j}(z)=a z+b$ is the unique affine mapping with $a>0$ and $b \in \mathbb{R}$ that maps $\bar{\square}_{j}$ to $\bar{\square}$. According to 2.11 , we would have

$$
f_{\square_{j}}\left|\frac{2\left(\mathcal{S} \mu_{\mathrm{per}}\right)^{\prime}}{\rho_{\mathbb{H}}}(z)\right|^{2} \frac{|d z|^{2}}{y}>\Sigma_{\mathcal{B}}^{2}+2 \varepsilon / 3, \quad \text { for all } \square_{j} \in \mathscr{G}_{n},
$$

when $n$ is large. However, this is not yet a contradiction since $\left\|\mathcal{S}^{\#} \mu_{\text {per }}\right\|_{\mathcal{B}(\mathbb{H})}$ might be greater than 1 .

For $z \in \bar{\square}_{j}$, let $h(z)=d_{\overline{\mathbb{H}}}\left(z, \partial \bar{\square}_{j}\right)$. Fix a number $S>0$ very large, but much smaller than $R=\log n$. We now modify $\mu_{\text {per }}$ on the set $W=\{z: h(z)<S\}$. More precisely, we define a new Beltrami coefficient

$$
\hat{\mu}_{\mathrm{per}}(z)= \begin{cases}(h(z) / S) \cdot \mu_{\mathrm{per}}, & z \in W \\ \mu_{\mathrm{per}}, & z \notin W .\end{cases}
$$

By making $S$ large, we can make $\left\|\mathcal{S}^{\#} \hat{\mu}_{\text {per }}\right\|_{\mathcal{B}(\mathbb{H})} \leq 1+\varepsilon / 3$. Furthermore, if $R \gg S$ is large, Lemma 2.2 and the isoperimetric property 2.10 guarantee that

$$
\left|\sigma_{[0,1]}^{2}\left(\mathcal{S}^{\#} \hat{\mu}_{\mathrm{per}}\right)-\sigma_{[0,1]}^{2}\left(\mathcal{S}^{\#} \mu_{\mathrm{per}}\right)\right| \leq \varepsilon / 3
$$

This contradicts the definition of $\Sigma_{\mathcal{B}}^{2}$ (we may divide $\hat{\mu}_{\text {per }}$ by $1+\varepsilon / 3$ ), so our initial assumption must have been wrong.

(ii) Conversely, suppose $b$ is a Bloch function with

$$
\|b\|_{\mathcal{B}(\mathbb{H})} \leq 1 \quad \text { and } \quad \sigma_{[0,1]}^{2}(b) \geq \Sigma^{2}-\varepsilon / 2
$$

Consider the $n$-adic grid $\mathscr{G}_{n}$. By the pigeon-hole principle, there exists an $n$-adic box $\square$ for which the integral in $(3.3)$ is at least $\Sigma^{2}-\varepsilon / 2$. Set $\nu=2 i \cdot \overline{b^{\prime}(\bar{z})} / \rho_{\overline{\mathbb{H}}}$ so that $\mathcal{S}^{\#} \nu=b+C$. Restricting $\nu$ to $\square$ and periodizing over $n$-adic boxes produces a Beltrami coefficient $\hat{\nu}_{\text {per }}$ which satisfies

$$
f_{\square_{j}}\left|\frac{2\left(\mathcal{S} \hat{\nu}_{\mathrm{per}}\right)^{\prime}}{\rho_{\mathbb{H}}}(z)\right|^{2} \frac{|d z|^{2}}{y}>\Sigma^{2}-\varepsilon, \quad \text { for all } \square_{j} \in \mathscr{G}_{n} .
$$

This completes the proof. 
Remark. To see that the periodization argument works for the norms

$$
\|b\|_{\mathcal{B} / \mathcal{B}_{0}, m}=\limsup _{|z| \rightarrow 1}\left(1-|z|^{2}\right)^{m}\left|b^{(m)}(z)\right|, \quad m \geq 2,
$$

it suffices to show that $\left.\mu \rightarrow \mid(\mathcal{S} \mu)^{(m+1)} / \rho_{\mathbb{H}}^{m}\right)(z) \mid$ satisfies a variant of Lemma 2.2 . We leave it to the reader to generalize the argument from [10, Section 4]. With a bit more work, one can show that the above argument is applicable to the Zygmund norm on the Bloch space. Here, one takes a Bloch function $b(z)$, forms

$$
f(z)=\int_{0}^{z} b(w) d w
$$

and then restricts to the unit circle, cf. [7, Theorems II.3.4 and VII.1.3]. A similar construction involving fractional integration endows the Bloch space with the $C^{\alpha}$ norms $(0<\alpha<1)$. More precisely, if $b \in \mathcal{B}$ then

$$
f(z)=\int_{\mathbb{D}} \frac{b(w)}{(1-z \bar{w})^{2-\alpha}}|d w|^{2}
$$

satisfies $\sup _{z \in \mathbb{D}}\left(1-|z|^{2}\right)^{1-\alpha}\left|f^{\prime}(z)\right| \leq C_{\alpha}\|b\|_{\mathcal{B}}$ and hence extends to a $C^{\alpha}$ function on the unit circle. In fact, if $b=P \mu$ with $\mu \in L^{\infty}(\mathbb{D})$ then Fubini's theorem shows that $f(z)=\int_{\mathbb{D}} \frac{\mu(w)}{(1-z \bar{w})^{2-\alpha}}|d w|^{2}$ from which the growth bound is clear. Further, the value of the $C^{\alpha}$ quotient $\frac{\left|f\left(z_{1}\right)-f\left(z_{2}\right)\right|}{\left|z_{1}-z_{2}\right|^{\alpha}}, z_{1}, z_{2} \in \mathbb{S}^{1}$ is determined (up to small error) by the values of $\mu$ in a neighbourhood of the Euclidean midpoint of the hyperbolic geodesic joining $z_{1}$ and $z_{2}$. The reader interested in working out the details can examine [23, Chapter 7] and [7, Theorem II.3.2].

These constructions lead to the constants $\Sigma_{\mathcal{B}, m}^{2}, \Sigma_{\mathrm{Z}}^{2}$ and $\Sigma_{C^{\alpha}}^{2}$. We note however that one can construct (equivalent) norms on $\mathcal{B} / \mathcal{B}_{0}$ which cannot be described as $\lim \sup _{|z| \rightarrow 1}$ of local behaviour. For these irregular norms, the periodization scheme does not work. 


\section{Coefficient estimates}

In this section, we prove Lemma 1.2 and give an explicit estimate for the quantity

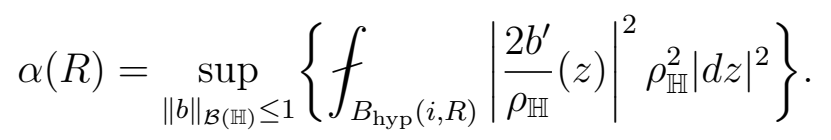

The proof of Lemma 1.2 uses the motif of geometry of averages which says that averages of high concentration control averages of low concentration, e.g. the $L^{\infty}$ norm controls the $L^{1}$ norm of a function.

Proof of Lemma 1.2. Suppose $b \in \mathcal{B}(\mathbb{H})$ is a Bloch function of norm 1. If we average

$$
f_{B_{\mathrm{hyp}}(z, R)}\left|\frac{2 b^{\prime}}{\rho_{\mathbb{H}}}(z)\right|^{2} \rho_{\mathbb{H}}^{2}|d z|^{2} \leq \alpha(R)
$$

over $z \in[0,1] \times[h, 1]$ with respect to the measure $|d z|^{2} / y$, we see that the quantity 3.2 is bounded by $\alpha(R)+\mathcal{O}_{R}(1 /|\log h|)$ as $h \rightarrow 0^{+}$. Thus, $\sigma_{[0,1]}^{2}(b) \leq \alpha(R)$ as desired.

We proceed to give a quantitative bound for $\alpha(R)$. For this purpose, we switch back to the unit disk and consider a Bloch function $b \in \mathcal{B}$ of norm 1. Let us expand $b^{\prime}$ as a power series:

$$
b^{\prime}(z)=q_{0}+q_{1} z+q_{2} z^{2}+q_{3} z^{3}+\cdots .
$$

To give a bound for

$$
\int_{|z| \leq r}\left|b^{\prime}(z)\right|^{2}|d z|^{2}=2 \pi \sum_{k=0}^{\infty} \frac{r^{2 k+2}}{2 k+2}\left|q_{k}\right|^{2},
$$

we need to estimate the coefficients $\left\{q_{k}\right\}$. Since $\|b\|_{\mathcal{B}} \leq 1$, Cauchy's estimates show:

$$
\left|q_{k}\right| \leq \frac{k+2}{2}\left(\frac{k+2}{k}\right)^{k / 2}, \quad k \geq 1 .
$$

We estimate the first few terms together, rather than one at a time. To do this, we will use Parseval's identity and the following general principle (see [2] or [5, Satz 
3.2.1]): To maximize a continuous and increasing functional of $\left|q_{0}\right|$ and $\left|q_{1}\right|$ in the unit ball of $\mathcal{B}$, it is enough to consider the special functions $b(z)=(3 / 4) \sqrt{3} S_{a}(z)^{2}$ where

$$
S_{a}(z)=\frac{z+a}{1+a z}, \quad a \in[0,1 / \sqrt{3}] .
$$

For these special functions,

$$
q_{0}=(3 / 2) \sqrt{3} a\left(1-a^{2}\right), \quad q_{1}=(3 / 2) \sqrt{3}\left(1-a^{2}\right)\left(1-3 a^{2}\right) .
$$

We remark that the parameter $a$ in our paper is denoted by $z_{0}$ in [5], also Bonk works with the Bloch function rather than its derivative, which explains the discrepancy in the factor of 2 between our $q_{1}$ and Bonk's $a_{2}$.

With this principle in mind, we give two estimates, one when $\left|q_{2}\right|$ is small, and another when $\left|q_{2}\right|$ is large:

Lemma 4.1. If $\left|q_{2}\right| \leq 2$, then for $r \leq 0.4$, the following inequality holds:

$$
\frac{r^{2}}{2}\left|q_{0}\right|^{2}+\frac{r^{4}}{4}\left|q_{1}\right|^{2}+\frac{r^{6}}{6}\left|q_{2}\right|^{2} \leq \frac{r^{2}}{2}+\frac{2}{3} r^{6} .
$$

Proof. Straightforward computations show that for all the special functions $S_{a}$,

$$
\frac{r^{2}}{2}\left|q_{0}\right|^{2}+\frac{r^{4}}{4}\left|q_{1}\right|^{2} \leq \frac{r^{2}}{2} .
$$

Hence, the above estimate is true for any function in the Bloch unit ball. Together with the assumption $\left|q_{2}\right| \leq 2$, this gives 4.3 .

Lemma 4.2. If $\left|q_{2}\right| \geq 2$, then for $r \leq 0.4$, we have

$$
\frac{r^{2}}{2}\left|q_{0}\right|^{2}+\frac{r^{4}}{4}\left|q_{1}\right|^{2}+\frac{r^{6}}{6}\left|q_{2}\right|^{2}+\frac{r^{8}}{8}\left|q_{3}\right|^{2} \leq \frac{r^{2}}{2}+\frac{17}{24} r^{6}+2.77 r^{8} .
$$

Proof. By Parseval's formula,

$$
\left|q_{0}\right|^{2}+\left|q_{1}\right|^{2} s^{2}+\left|q_{2}\right|^{2} s^{4}+\left|q_{3}\right|^{2} s^{6}+\cdots \leq \frac{1}{\left(1-s^{2}\right)^{2}}, \quad s \in[0,1] .
$$

In particular,

$$
\left|q_{3}\right|^{2} s^{6} \leq \frac{1}{\left(1-s^{2}\right)^{2}}-4 s^{4}
$$


since $\left|q_{2}\right| \geq 2$ by assumption. The choice $s^{2}=0.58$ gives $\left|q_{3}\right|^{2} \leq 22.16$ or

$$
\frac{r^{8}}{8}\left|q_{3}\right|^{2} \leq 2.77 r^{8}
$$

It remains to show that

$$
\frac{r^{2}}{2}\left|q_{0}\right|^{2}+\frac{r^{4}}{4}\left|q_{1}\right|^{2}+\frac{r^{6}}{6}\left|q_{2}\right|^{2} \leq \frac{r^{2}}{2}+\frac{17}{24} r^{6} .
$$

For this purpose, consider the function

$$
\phi(z)=\frac{1-\tau}{2}\left(b^{\prime}(\sqrt{\tau} z)+b^{\prime}(-\sqrt{\tau} z)\right)=(1-\tau) q_{0}+(1-\tau) \tau q_{2} z^{2}+\cdots,
$$

where $\tau$ is an auxiliary parameter in $[0,1]$. By construction, $|\phi(z)| \leq 1$ in the unit disk. Applying the Schwarz lemma to $\phi(\sqrt{z})$ gives

$$
(1-\tau) \tau\left|q_{2}\right| \leq 1-(1-\tau)^{2}\left|q_{0}\right|^{2} .
$$

Maximizing over $\tau \in[0,1]$, we obtain

$$
\left|q_{2}\right| \leq 2+2 \sqrt{1-\left|q_{0}\right|^{2}} .
$$

Therefore to prove 4.5), we have to show that the inequality

$$
\frac{r^{2}}{2}\left|q_{0}\right|^{2}+\frac{r^{4}}{4}\left|q_{1}\right|^{2}+\frac{r^{6}}{6}\left(2+2 \sqrt{1-\left|q_{0}\right|^{2}}\right)^{2} \leq \frac{r^{2}}{2}+\frac{17}{24} r^{6}, \quad r \leq 0.4,
$$

is valid for all $q_{0}, q_{1}$ of the form 4.2 , with $a \in[0,1)$. Routine computations show that this is indeed the case. We leave the details to the reader.

Lemma 4.3. If $r=0.4$ then

$$
\frac{\left(1-r^{2}\right)}{\pi r^{2}} \int_{|z| \leq r}\left|b^{\prime}(z)\right|^{2}|d z|^{2} \leq 0.8998 .
$$

Proof. If $\left|q_{2}\right| \leq 2$, then from Lemma 4.1 and Cauchy's estimates 4.1],

$$
\sum_{k=0}^{\infty} \frac{r^{2 k+2}}{2 k+2}\left|q_{k}\right|^{2} \leq \frac{r^{2}}{2}+\frac{2}{3} r^{6}+\sum_{k=3}^{\infty} \frac{r^{2 k+2}}{2 k+2}\left(\frac{k+2}{2}\right)^{2}\left(\frac{k+2}{k}\right)^{k},
$$


while if $\left|q_{2}\right| \geq 2$, Lemma 4.2 gives

$$
\sum_{k=0}^{\infty} \frac{r^{2 k+2}}{2 k+2}\left|q_{k}\right|^{2} \leq \frac{r^{2}}{2}+\frac{17}{24} r^{6}+2.77 r^{8}+\sum_{k=4}^{\infty} \frac{r^{2 k+2}}{2 k+2}\left(\frac{k+2}{2}\right)^{2}\left(\frac{k+2}{k}\right)^{k} .
$$

Plugging in the value $r=0.4$, numerical computations show that in either case, the left hand side does note exceed 0.8998 .

Since the hyperbolic area of the ball $B(0, r)$ is

$$
\int_{|z| \leq r} \rho^{2}|d z|^{2}=2 \pi \int_{0}^{r} \frac{4 s}{\left(1-s^{2}\right)^{2}} d s=4 \pi \frac{r^{2}}{1-r^{2}},
$$

we see that Lemma 4.3 is equivalent to the statement

$$
\alpha(\eta(0.4))<0.8998
$$

where $\eta(0.4)$ is the hyperbolic radius of the ball $\{z:|z| \leq 0.4\}$. This shows $\Sigma_{\mathcal{B}}^{2}<0.9$, which completes the proof of Theorem 1.2 .

\section{References}

[1] K. Astala, O. Ivrii, A. Perälä, I. Prause, Asymptotic variance of the Beurling transform, Geom. Funct. Anal. 25 (2015), no. 6, 1647-1687.

[2] F. G. Avhadiev, I. R. Kayumov, Estimates for bloch functions and their generalization, Complex Variables and Elliptic Equations 29 (1996), no. 3, 193-201.

[3] R. Bañuelos, Brownian motion and Area Functions, Indiana Univ. Math. J. 35 (1986), no. 3, 643-668.

[4] R. Bañuelos, C. N. Moore, Mean growth of Bloch functions and Makarov's law of the iterated logarithm, Proc. Amer. Math. Soc. 112 (1991), 851-854.

[5] M. Bonk, Extremalprobleme bei Bloch-funktionen, PhD Thesis, TU Braunschweig (1988). 
[6] F. P. Gardiner, N. Lakic, Quasiconformal Teichmüller theory, Mathematical Surveys and Monographs 76, Amer. Math. Soc., 2000.

[7] J. B. Garnett, D. E. Marshall, Harmonic Measure, New Mathematical Monographs 2, Cambridge University Press, 2005.

[8] H. Hedenmalm, Bloch functions, asymptotic variance and geometric zero packing, preprint, 2016. arXiv:1602.03358

[9] H. Hedenmalm, I. R. Kayumov, On the Makarov law of the iterated logarithm, Proc. Amer. Math. Soc. 135 (2007), 2235-2248.

[10] O. Ivrii, Quasicircles of dimension 1+k do not exist, preprint, 2016. arXiv:1511.07240.

[11] O. Ivrii, On Makarov's principle in conformal mapping, preprint, 2016. arXiv:1604.05619.

[12] I. R. Kayumov, The law of the iterated logarithm for locally univalent functions, Ann. Acad. Sci. Fenn. Math. 27 (2002), no. 2, 357-364.

[13] T. H. N. Le, M. Zinsmeister, On Minkowski dimension of Jordan curves, Ann. Acad. Sci. Fenn. Math. 39 (2014), no. 2, 787-810.

[14] T. Lyons, A synthetic proof of Makarov's law of the iterated logarithm, Bull. London Math. Soc. 22 (1990), 159-162.

[15] N. G. Makarov, On the distortion of boundary sets under conformal mappings, Proc. London Math. Soc. 51 (1985), 369-384.

[16] N. G. Makarov, Conformal mapping and Hausdorff measures, Ark. Mat. 25 (1987), no. 1, 41-89.

[17] N. G. Makarov, Probability methods in the theory of conformal mappings, (Russian) Algebra i Analiz 1 (1989), no. 1, 3-59; translation in Leningrad Math. J. 1 (1990), no. $1,1-56$. 
[18] C. T. McMullen, Thermodynamics, dimension and the Weil-Petersson metric, Invent. Math. 173 (2008), no. 2, 365-425.

[19] A. Perälä, On the optimal constant for the Bergman projection onto the Bloch space, Ann. Acad. Sci. Fenn. Math. 37 (2012), no. 1, 245-249.

[20] C. Pommerenke, The growth of the derivative of a univalent function, in: The Bieberbach Conjecture (West Lafayette, Ind. 1985), Mathematical Surveys and Monographs 21, Amer. Math. Soc., 1986, 143-152.

[21] C. Pommerenke, Boundary behaviour of conformal maps, Grundlehren der Mathematischen Wissenschaften 299, Springer-Verlag, 1992.

[22] F. Przytycki, On the law of iterated logarithm for Bloch functions, Studia Math. 39 (1989), 145-154.

[23] K. Zhu, Spaces of Holomorphic Functions in the Unit Ball, Graduate Texts in Mathematics 226, Springer, 2005. 